\title{
Near-Field Cross Section Imaging of Wideband Millimeter Wave
}

\author{
Yingzhi Kan , Yongfeng Zhu and Qiang Fu \\ School of Electronic Science and Engineering, National University of Defense Technology, Hunan, China
}

\begin{abstract}
Near-field millimeter wave imaging has been a hot topic recent years for its importance applications in the area of anti-terrorism. The penetrating characteristic of millimeter wave is of significant importance to security, such as the concealed weapons detection, ground-penetrating radar imaging, through-barrier imaging and so on. Cross section imaging is a basic aspect for near-field millimeter wave imaging, which includes antenna array distribution and wideband signal processing. This paper utilizes back projection method in space area to realize ultra-band nearfield cross section imaging. We induce two dimensional direction integral formulas to obtain the reconstruction image of the near-field imaging area, and the simulation results validate the effectiveness of this imaging algorithm.
\end{abstract}

\section{Introduction}

The demand for millimeter wave imaging techniques increases in the field of nondestructive testing (NDT) or in security applications since the threat of terrorism attacks is increasing these years[1, 2]. The security detection in public is to detect the threatened weapons under person's clothes or dangerous objects in the luggage. The X-ray detection system and the metal detectors are common methods for security check. These systems have their own advantages and disadvantages. Metal detector can only detect metal objects, but not be useful for some modern weapons such as plastic or chinaware gun, and liquid bombshell. On the other hand, some metal commodity may be detected as metal weapons to result in false alarms. To decrease false alarms, the imaging systems like the X-ray imaging system and the millimeter wave imaging system can both be utilized to overcome the defects of metal detectors. Comparing with X-ray which is dangerous when illuminate person, the millimeter wave system transmits low power with no harm to person. Besides, the millimeter wave system can penetrate clothes and luggage, which make it a prospective application in security area.

Near-field millimeter wave imaging system, could realize coronal plane imaging, cross section imaging and three dimensional imaging $[3,4]$. Coronal plane imaging and cross section imaging are basic problems for studying three dimensional imaging. Coronal plane imaging achieves two dimensional resolutions by scan a rectangular planar aperture. Cross section imaging achieves two dimensional resolutions by a linear antenna array in one dimension and a wideband signal in another dimension. In this paper, we mainly discuss cross section imaging.
Near-field cross section imaging mode transmits wideband millimeter wave along a linear array [5]. To improve resolution, we can expand the linear antenna array to enhance the cross resolution, and increase the wideband of transmitted signal to enhance the range resolution. Here, we adopt stepped frequency wideband signal. As for near-field millimeter imaging system, the carrier frequency is high which can achieve ultra-band transmitted signal to reach millimeter resolution. Common cross section imaging algorithms, based on spectral decomposition theory, utilize space filtering and interpolation to obtain effective two dimensional space spectrum data to implement Fourier two dimensional imaging. In the imaging process, the main imaging error is introduced by interpolation which directly affects the accuracy and efficiency of the images. In this paper, we consider the ultra-band signal and small imaging scene, so that, it is not available to utilize interpolation algorithm since the wideband is not sufficient used in the means of interpolation in that case. To avoid interpolation, we introduce the back projection algorithm [6] to directly calculate two dimensional integral of the recorded echo signal in the form of frequency to reconstruct near-field image. The paper derives the formula of direct two dimensional integral and simulation verifies the effectiveness of the algorithm. This work is important to extend to study the three dimensional near-field millimeter wave imaging.

\section{Algorithm description}

Cross section image reflects the scattering distribution along the cross direction and the range direction. We set cross direction as $\mathrm{x}$ direction and range direction as $\mathrm{z}$ direction, and the returned scattered data is recorded by an two dimensional matrix. 


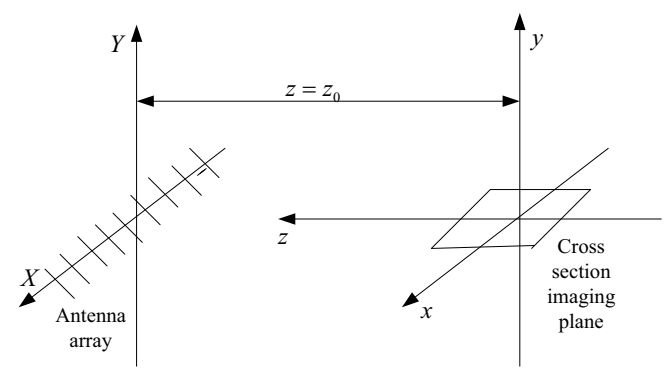

Figure 1. Geometry of cross section imaging

The basic imaging configuration is shown in Figure 1. The imaging transceiver is scanned, mechanically or electronically along a linear array aperture. At each sampling point in the aperture, a wide beam-width transmitting antenna is used to emit the millimeter wave signal, which is generally swept over a wide frequency bandwidth. The transmitting and receiving antennas are generally identical in construction, placed in close proximity to each other, and scanned together in a simultaneous source-receiver scanning configuration. The antenna placement is referred to as a quasi-monostatic configuration. The antennas are distinct, but can be considered to be a single antenna located at the midpoint between the two antenna phase centers. This approximation is valid for target ranges significantly larger than the antenna spacing.

The transceiver moves along $X$ direction step by step, which transmits stepped frequency signal and records the scattered echo data in frequency domain. This measurement process is repeated at each sampling point. After ergodic all the sampling points in $X$ direction, the system records a measurement matrix, of which each element represents a scattered data at a certain space position and a certain frequency. The destination of cross section imaging is to reconstruct scattering distribution of target in the $x-z$ plane according to the recorded data.

Generally, cross section imaging algorithms are based on spectral decomposition theory. The electric field distributed in $\mathrm{X}-\mathrm{Z}$ plane can be obtained according to the wave spectrum transferring relationship, which is equal to the scattering distribution function of the targets.

The classical algorithm has been detailed described in [5].The imaging algorithm mainly concludes a onedimensional FFT, a space filtering, a two-dimensional interpolation and a two-dimensional IFFT. The imaging precision is affected by the interpolation process.

\subsection{Direct integral algorithm}

For near-field wideband millimeter wave imaging, when the imaging scene is small and the range between the target and the antenna array is short, the interpolation process become difficult to achieve to assure interpolation accuracy. To overcome this disadvantage we introduce two dimensional direct imaging methods.

Suppose targets located in $x-z$ plane and measuring antenna located in $X$ direction. The $X$ axis parallels to $x$ axis and the perpendicular range between $x$ and $X$ is $z_{0}$. The length of the antenna array is $L_{x}$.

The recorded scattered data $E(X, k)$ is a twodimensional matrix, which can be denoted as

$$
E(X, k)=\iint \sigma(x, z) \exp (-j k \cdot r) d x d z
$$

Where

$$
\begin{aligned}
& r=\sqrt{(x-X)^{2}+\left(z-z_{0}\right)^{2}} \\
& k=4 \pi f / c \\
& k^{2}=k_{x}^{2}+k_{z}^{2}
\end{aligned}
$$

Here, $c$ is the speed of light, $f$ is temporal frequency, $k$ is the wavenumber frequency, and $k_{x}, k_{z}$ are the three components of $k$ along $x, z$ direction respectively. The aim of imaging process is to reconstruct the 2-D reflectivity function $\sigma(x, z)$ from the recorded scattered data $E(X, k)$.

Then, we can get

$$
\begin{aligned}
\sigma(x, z)= & \iint E\left(k_{x}, k_{z}\right) \\
& \cdot \exp \left[j\left(k_{x} \cdot(x-X)+k_{z} \cdot\left(z-z_{0}\right)\right] d k_{x} d k_{z}\right.
\end{aligned}
$$

Let

$$
\begin{aligned}
& k_{x}=k \cdot \cos \theta=k \cdot \frac{x-X}{r} \\
& =k \cdot \frac{x-X}{\sqrt{\left(z-z_{0}\right)^{2}+(x-X)^{2}}} \\
& k_{z}=k \cdot \sin \theta=k \cdot \frac{z-z_{0}}{r} \\
& =k \cdot \frac{z-z_{0}}{\sqrt{\left(z-z_{0}\right)^{2}+(x-X)^{2}}}
\end{aligned}
$$

We obtain

$$
\begin{aligned}
\sigma(x, z)= & \iint E\left(k \cdot \frac{x-X}{r}, k \cdot \frac{z-z_{0}}{r}\right) \\
& \cdot \exp (j k \cdot r) d k_{x} d k_{z} \\
= & \iint F\left(k \cdot \frac{x-X}{r}, k \cdot \frac{z_{0}-Z}{r}\right) \\
& \exp (j k \cdot r) \cdot\left|\frac{\partial\left(k_{x}, k_{z}\right)}{\partial(k, x)}\right| d k d x
\end{aligned}
$$

Let

$$
J=\left|\frac{\partial\left(k_{x}, k_{z}\right)}{\partial(k, x)}\right|=\left|\begin{array}{ll}
\frac{\partial k_{x}}{\partial k} & \frac{\partial k_{x}}{\partial x} \\
\frac{\partial k_{z}}{\partial k} & \frac{\partial k_{z}}{\partial x}
\end{array}\right|=k \cdot \frac{\left|z-z_{0}\right|}{r^{2}}
$$

Then

$$
\begin{gathered}
\sigma(x, z)=\iint E\left(k \cdot \frac{x-X}{r}, k \cdot \frac{z-z_{0}}{r}\right) \exp (j k \cdot r) \\
\cdot k \cdot \frac{\left|z-z_{0}\right|}{\left(z-z_{0}\right)^{2}+(x-X)^{2}} d k d x
\end{gathered}
$$

$\mathrm{Or}$

$$
\begin{aligned}
\sigma(x, z)=\iint & E(X, k) \exp (j k \cdot r) \\
& \cdot k \cdot \frac{\left|z-z_{0}\right|}{\left(z-z_{0}\right)^{2}+(x-X)^{2}} d k d x
\end{aligned}
$$


That is the direct two-dimensional integral reconstruction algorithm.

\subsection{2-D resolution analysis}

Image resolution reflects the details of reconstructed image, which is a first criterion and shows the accuracy of the imaging system. The ability of high resolution is quite important to detect concealed weapons. Image resolution can be considered as the ability to distinguish two scatters located very close to each other. So the resolution along cross direction is in [5].

$$
\delta_{x}=\frac{\lambda}{2 L_{x}} \sqrt{z_{0}^{2}+L_{x}^{2} / 4}
$$

Generally, in far-field the range resolution is decided by the signal bandwidth. However in the near-field situation, since the range between the target and antenna cannot approximate, the wavefront is spherical, and the cross range resolution is decided by the occupied $k_{z}$. That is

$$
\delta_{z}=\pi /\left(K_{z \max }-K_{z \min }\right)
$$

The expression shows that the cross resolution of the near-field millimetre wave system is decided by the signal frequency, the perpendicular distance between the scanning plane and the scatters, and the scan size. The system parameters can be chosen by considering the image resolution requirement.

\section{Simulation experiment}

The transceiver antenna scans along a linear array of $(-15 \mathrm{~cm}, 15 \mathrm{~cm})$, at each sampling point, the antenna transmits stepped frequency signal with $10 \mathrm{GHz} \sim 40 \mathrm{GHz}$ bandwidth. The frequency interval is $\Delta f=100 \mathrm{MHz}$, the antenna sampling interval is $\Delta x=3.75 \mathrm{~mm}$. The area to be imaged is $20 \mathrm{~cm} \times 20 \mathrm{~cm}$, and the perpendicular distance to the antenna plane is $30 \mathrm{~cm}$. There are three scatters located in the imaging area, as Figure 2.

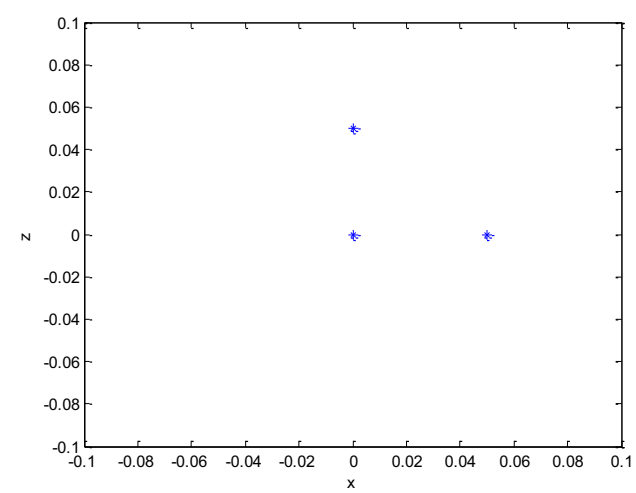

Figure 2. Simulated targets

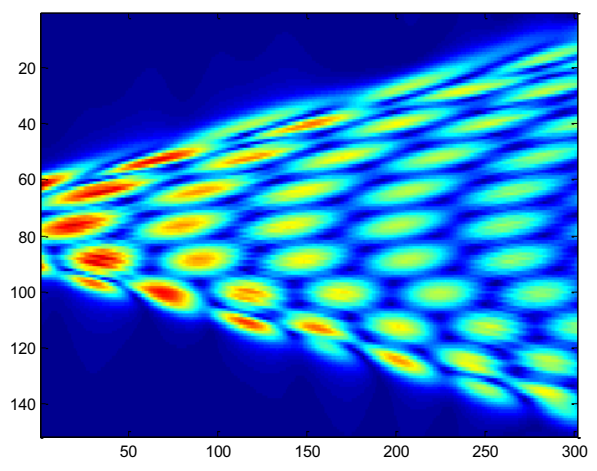

Figure 3. 2-D space spectrum

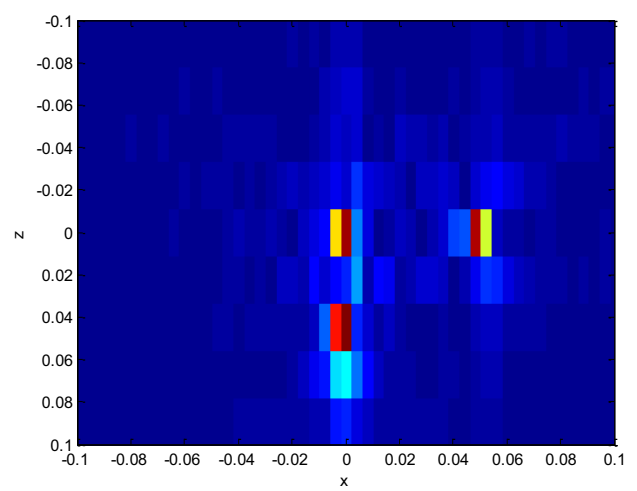

Figure 4. Interpolation based imaging result

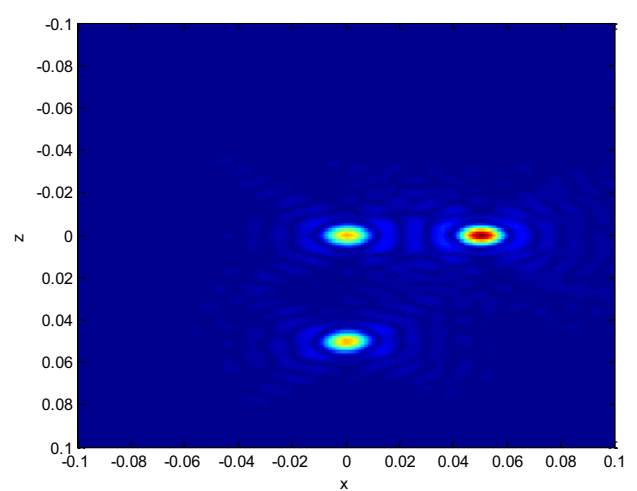

Figure 5. Direct two-dimensional integral results

The imaging results are shown from Figure 3 to Figure 5. Figure 3 is the 2-D space spectrum of the scattered data and it is clear that the spectrum is not uniform. Figure 4 is the imaging result of the conventional method. The cross section imaging result of the proposed method is as Figure 5. In Figure 5, the three point scatters are focused well and are located at the exact position. It is very obvious in the image and no calibration is required comparing to the original scene. Compared with the twodimensional interpolation method, the proposed direct integral method is simple and direct. The imaging accuracy is decided directly by the sampling returned signal, not affected by the signal processing method. And because of the small imaging scene, the direct twodimensional integral method is of high calculation efficiency. The experiment demonstrates the direct twodimensional method is available for the near-field millimeter wave imaging. 
As for the interpolation method, in the situation of near-field and small imaging scene, is not appropriate. The linear antenna array is short resulting in the twodimensional space spectrum in $\mathrm{k}$-space is curving. These make the two-dimensional interpolation difficult to accurately implement. Also, the range resolution is affected by the interpolation result since the resolution is decided by the span of $K_{z}$. The span of $K_{z}$ is limited when the curvature of space spectrum is severe which directly restrict the range resolution and lower the bandwidth lower utilization rate. The direct twodimensional integral method avoids interpolation and can make the full use of the wide bandwidth to realize high resolution imaging.

\section{Conclusions}

The paper discusses the wideband millimeter wave nearfield cross section imaging. Since the two-dimensional Fourier method needs to resolve the interpolation problem, and in the certain case of near-field small imaging scene the interpolation problem become more difficult to deal with. To avoid interpolation and simplify the imaging process, we induce the back projection formula to achieve direct two-dimensional integral imaging of cross section, and analyze the resolution of the two dimensions. We set an imaging scene and related parameters to simulate the imaging progress of near-field cross section imaging. The simulation result is focused well to demonstrate the effectiveness of the proposed algorithm. And since the imaging scene is small the calculation is simple. It is an efficient imaging algorithm for near-field cross section imaging.

\section{References}

1. Frank Gumbmann, Phat Tran, Lorenz-Peter Schmidt. Sparse Linear Array Design for a Short Range Imaging Radar. 2009 EuMA

2. M. Ravan, Reza K. Amimeh, and Natalia K. Nikolova. Two-dimensional near-field microwave holography. INVERSE PROBLEMS,26, 2010.

3. David Sheen, Douglas McMakin, and Thomas Hall. Near-field Three-dimensional Radar Imaging Techniques and Applications. APPLIED OPTICS, 49(19), 2010.

4. ZHANG Yansheng, YANG Jungang, AN Wei. Single frequency two-dimensional synthetic aperture imaging system and imaging algorithm. Journal of National University of Defense Technology, 36(4), 2014.

5. Zhang Xilin, li Nanjing, Hu Chufeng, Li Ping. Radar Target Cross Section Characteristics Test and Imaging Diagnosis. China Astronautic Publishing House, 2009.

6. Huang Peikang, Yin Hongcheng, Xu Xiaojian. Radar Targets Characteristics. Publishing House of Electronics Industry, 2010. 